\begin{tabular}{|c|c|c|c|c|c|c|c|}
\hline Gene & Entrez ID & GS & $P(\mathrm{GS})$ & MM & $P(\mathrm{MM})$ & $\log _{2}$ FC (SMA vs CM) & FDR \\
\hline МАР3К20 & 51776 & -0.485664 & .000623 & 0.823353624 & 2.15E-12 & 1.022307 & 0.004612 \\
\hline $\operatorname{ccs}$ & 9973 & -0.441517 & .002129 & 0.896535459 & 3.66E-17 & 0.952507 & 0.004764 \\
\hline TRIM10 & 10107 & -0.424089 & .003312 & 0.956470241 & 3.65E-25 & 1.713361 & 0.000648 \\
\hline MX11 & 4601 & -0.421417 & .003537 & 0.683420545 & $1.66 \mathrm{E}-07$ & 1.815240 & 0.000738 \\
\hline EPB41 & 2035 & -0.402531 & .005548 & 0.863053376 & 1.22E-14 & 1.780446 & 0.000399 \\
\hline
\end{tabular}

Abbreviations: CM, cerebral malaria; FC, fold change; FDR, false discovery rate; GS, gene significance; MM, module membership; SMA, severe malarial anemia.

Received 9 February 2019; editorial decision 12 March 2019; accepted 13 March 2019 ; published online March 14, 2019.

Correspondence: K. Li, PhD, Department of Blood Transfusion, Tongji Hospital, Tongji Medical College, Huazhong University of Science and Technology, No.1095 Jiefang Avenue, Hankou, Wuhan 430030, P. R. China (likelff1@hust.edu.cn).

The Journal of Infectious Diseases ${ }^{\circledR} \quad$ 2019;220:540-2 (C) The Author(s) 2019. Published by Oxford University Press for the Infectious Diseases Society of America. All rights reserved. For permissions, e-mail: journals.permissions@oup.com. D0I: 10.1093/infdis/jiz120

\section{Reply to Liu et al}

To THE EDITOR-We appreciate the analysis by Liu et al [1], which applied weighted gene co-expression network analysis (WGCNA) to the published transcriptomic dataset of cerebral malaria $(\mathrm{CM})$ and severe malarial anemia (SMA). We agree that WGCNA [2] is a powerful, data-driven approach for interrogating transcription profiles that can reveal important relationships not seen with differential gene expression analysis. With this approach, Liu et al [1] showed that heme metabolism was the most significantly enriched pathway among genes that were differentially expressed between clinical groups, which is consistent with our original analysis [3] that showed heme biosynthesis, iron homeostasis, and erythrocyte differentiation pathways to differ between CM and SMA. Also, of the 5 hub genes identified by their analysis, TRIM10, MXI1, and $E P B 41$, were differentially expressed between CM and SMA at a false discovery rate $<5 \%$ in our analysis [3].

However, Liu et al [1] noted inconsistencies between their results and our original findings, which can be explained by important differences between the 2 analyses. In contrast to the unadjusted analyses by Liu et al [1], we controlled for parasite biomass and sickle cell anemia by including plasma histidine-rich protein 2 (HRP2) levels and the presence of the HbSS genotype, respectively, as covariates in our analyses. Parasite biomass has been shown to drive a large proportion of the host transcriptional changes during severe malaria [4] and could potentially confound the differences between CM and SMA. Sickle cell anemia contributes to anemia independently of malaria and affects malaria mortality differently depending on the setting $[5,6]$. The contributions of parasite biomass and sickle cell anemia to transcriptomic variation can be seen in the principal components analyses in our original study [3] and when included in WGCNA module-trait relationship plots (Figure 1). The increased transcriptional activity in SMA observed by Liu et al [1] was likely due to differences in gene expression and/or cellular composition resulting from sickle cell anemia, as HbSS exclusion or adjustment reduces the number of differentially expressed genes for SMA relative to controls (Table 1).

Lastly, in our original study, we examined differences in biological pathways between CM and SMA using 2 approaches. The first approach applied differentially expressed genes that met the thresholds of $\log _{2}$ fold change $\geq 0.585$ and false discovery rate $<10 \%$ to Ingenuity pathways analysis. However, the second approach employed feature set enrichment analysis using tmod [7], which uses a list of genes ordered by minimum significant difference without applying fold change or significance thresholds and therefore can capture potentially important genes with lower fold changes.
Since publishing our article, we have developed a publicly accessible dashboard (https://malariasystems.shinyapps. io/CM_SMA/) for exploring the dataset in depth that includes both tmod and WGCNA.

\section{Notes}

Financial support. This work was supported by the National Institute of Allergy and Infectious Diseases (grant number K08AI125682 to T. M. T).

Potential conflicts of interest. Both authors: No reported conflicts. Both authors have submitted the ICMJE Form for Disclosure of Potential Conflicts of Interest. Conflicts that the editors consider relevant to the content of the manuscript have been disclosed.

Srinivas Nallandhighal ${ }^{1}$ and Tuan M. Tran ${ }^{2,3, \odot}$

'Department of Urology, University of Michigan, Ann Arbor Michigan, ${ }^{2}$ Division of Infectious Diseases, Department of Medicine, and ${ }^{3}$ Ryan White Center for Pediatric Infectious Disease and Global Health, Indiana University School of Medicine, Indianapolis, Indiana

\section{References}

1. Liu FF, Hu F, Xiong W, et al. New insights in transcriptomic differences between two major malaria phenotypes. J Infect Dis 2019.

2. Langfelder P, Horvath S. Fast R functions for robust correlations and hierarchical clustering. J Stat Softw 2012; 46:pii: i11.

3. Nallandhighal S, Park GS, Ho YY, Opoka RO, John CC, Tran TM. Whole-blood transcriptional signatures composed of erythropoietic and NRF2-regulated genes differ between cerebral malaria and severe malarial anemia. J Infect Dis 2019; 219:154-64. 


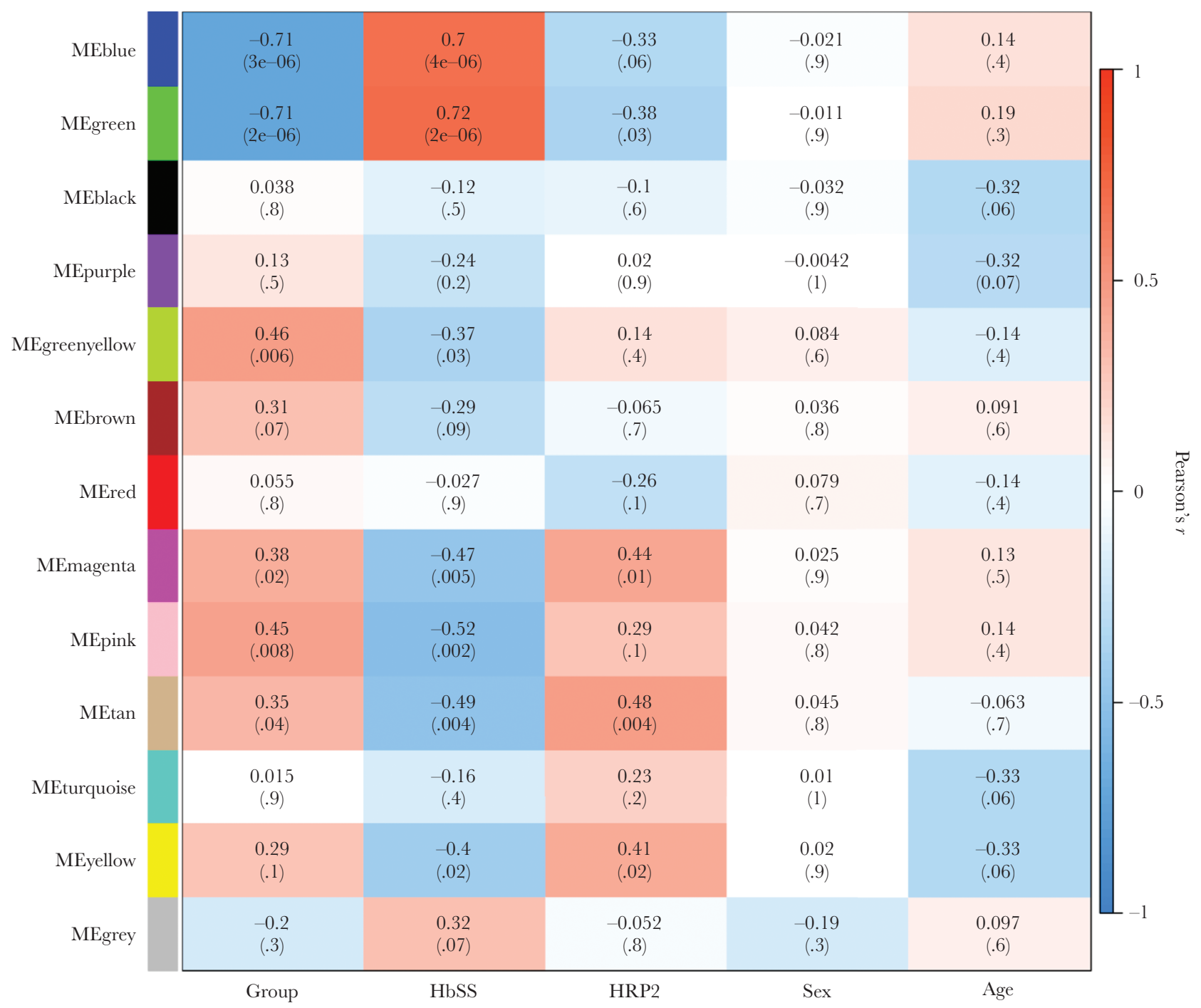

Figure 1. Module-trait relationship plot. Modules were generated by weighted gene co-expression network analysis using the top 15\% most variably expressed genes by median absolute deviation filtering ( $\mathrm{nGenes}=4997$ ). The 13 most significant modules containing $>100$ genes are shown as rows and traits are shown as columns. Severe malarial anemia is the reference group. Numbers and color scale represent Pearson $r$ with corresponding unadjusted asymptotic $P$ values shown in parentheses. Abbreviations: HbSS, sickle cell anemia; HRP2, histidine-rich protein 2.

\section{Table 1. Number of Differentially Expressed Genes for Each Comparison}

\begin{tabular}{lcccccc}
\hline & & \multicolumn{5}{c}{ Nallandhighal et al } \\
\cline { 3 - 7 } Comparison & Liu et al, Unadjusted & Unadjusted & HbSS Children Excluded & HbSS Adjusted & HRP2 Adjusted & All Adjusted \\
\hline CM vs CC & 2531 & 2971 & 3065 & 3123 & 686 & 3762 \\
SMA vs CC & 5199 & 4106 & 1919 & 373 & 1117 & 873 \\
CM vs SMA & 1323 & 1647 & 398 & 377 & 332 \\
\hline
\end{tabular}

Differential gene expression was performed using the empirical Bayes moderated $t$ test in the limma package with data obtained from Nallandhighal et al. [3]. For this table, the threshold for determining the number of differentially expressed genes was a false discovery rate $<10 \%$ with no fold-change threshold. Differences between unadjusted models may be due to differences in analysis package versions, prefiltering parameters, and/or the definition of unique transcripts or genes.

Abbreviations: CC, community children; CM, cerebral malaria; HbSS, sickle cell anemia; HRP2, histidine-rich protein 2; SMA, severe malarial anemia.

${ }^{a} \mathrm{HbSS}, \mathrm{HRP} 2$, and microarray processing batch were included as covariates in the original analysis, Nallandhighal et al [3]. 
4. Lee HJ, Georgiadou A, Walther M, et al. Integrated pathogen load and dual transcriptome analysis of systemic host-pathogen interactions in severe malaria. Sci Transl Med 2018; 10:pii: eaar3619.

5. McAuley CF, Webb C, Makani J, et al. High mortality from Plasmodium falciparum malaria in children living with sickle cell anemia on the coast of Kenya. Blood 2010; 116: 1663-8.

6. Opoka RO, Bangirana P, Idro R, Shabani E, Namazzi R, John CC. Lack of mortality in 22 children with sickle cell anemia and severe malarial anemia. Pediatr Blood Cancer 2018; 65:doi: 10.1002/pbc.26745.

7. Weiner J III, Domaszewska T. tmod: an $\mathrm{R}$ package for general and multivariate enrichment analysis. PeerJ Preprints 2016; 4:e2420v1.

Received 11 March 2019; editorial decision 12 March 2019; accepted 13 March 2019 ; published online March 14, 2019.

Correspondence: T. M. Tran, MD, PhD, c1044 West Walnut Street, Indianapolis, IN 46202. (tuantran@iu.edu).

The Journal of Infectious Diseases ${ }^{\circledR} \quad$ 2019;220:542-4

(C) The Author(s) 2019. Published by Oxford University Press for the Infectious Diseases Society of America. All rights reserved.

For permissions, e-mail: journals.permissions@oup.com. DOI: 10.1093/infdis/jiz121 